\title{
Platelet-to-Lymphocyte Ratio at Admission as a Predictor of In-Hospital and Long-Term Outcomes in Patients With ST-Segment Elevation Myocardial Infarction Undergoing Primary Percutaneous Coronary Intervention: A Systematic Review and Meta-Analysis
}

\author{
Herick Alvenus Willim ${ }^{\mathrm{a}, \mathrm{d}}$, Joan Carmen Harianto ${ }^{\mathrm{b}}$, Harie Cipta ${ }^{\mathrm{c}}$
}

\begin{abstract}
Background: ST-segment elevation myocardial infarction (STEMI) is the most severe form of acute coronary syndrome (ACS) which is associated with significant adverse outcomes. Platelet-to-lymphocyte ratio (PLR) is a novel inflammatory biomarker that has been used as a predictor of various cardiovascular diseases, including ACS. This meta-analysis aimed to investigate the prognostic value of PLR as a predictor of in-hospital and long-term outcomes in patients with STE$\mathrm{MI}$ undergoing primary percutaneous coronary intervention (PCI).
\end{abstract}

Methods: We performed a comprehensive systematic literature search in the databases of PubMed, ScienceDirect, Cochrane Library, and ProQuest for eligible studies. The primary outcomes were major adverse cardiac events (MACEs) and mortality, both in-hospital and long-term follow-up. The outcomes were compared between patients with high and low admission PLR. The quality assessment was conducted using the Newcastle-Ottawa scale. Review Manager 5.3 was used to perform the meta-analysis.

Results: Six cohort studies involving 4,289 STEMI patients undergoing primary PCI were included in this meta-analysis. The pooled analysis showed that a high PLR at admission was associated with increased in-hospital MACE (odds ratio $(\mathrm{OR})=1.94,95 \%$ confidence interval $(\mathrm{CI})=1.56-2.40, \mathrm{P}<0.00001, \mathrm{I}^{2}=45 \%$ ) and in-hospital mortality $\left(\mathrm{OR}=2.07 ; 95 \% \mathrm{CI}=1.53-2.80 ; \mathrm{P}<0.00001 ; \mathrm{I}^{2}=50 \%\right)$,

Manuscript submitted January 16, 2021, accepted January 23, 2021

Published online February 23, 2021

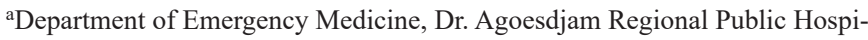
tal, Ketapang, West Kalimantan, Indonesia

${ }^{b}$ Faculty of Medicine, Tanjungpura University, Pontianak, West Kalimantan, Indonesia

'Department of Cardiology and Vascular Medicine, Dr. Agoesdjam Regional Public Hospital, Ketapang, West Kalimantan, Indonesia

${ }^{\mathrm{d} C}$ Corresponding Author: Herick Alvenus Willim, Department of Emergency Medicine, Dr. Agoesdjam Regional Public Hospital, Ketapang, West Kalimantan, Indonesia. Email: herick_alvenus@yahoo.co.id

doi: https://doi.org/10.14740/cr1219 as well as increased long-term MACE $(\mathrm{OR}=1.98 ; 95 \% \mathrm{CI}=1.31$ $\left.3.00 ; \mathrm{P}=0.001 ; \mathrm{I}^{2}=72 \%\right)$ and long-term mortality $(\mathrm{OR}=2.79 ; 95 \%$ $\mathrm{CI}=1.45-5.36 ; \mathrm{P}=0.002 ; \mathrm{I}^{2}=83 \%$ ).

Conclusions: In patients with STEMI undergoing primary PCI, a high PLR at admission predicts in-hospital MACE and mortality along with long-term MACE and mortality.

Keywords: Platelet-to-lymphocyte ratio; Major adverse cardiac event; Mortality; ST-segment elevation myocardial infarction; Percutaneous coronary intervention

\section{Introduction}

Coronary heart disease (CHD) is recognized as the leading cause of morbidity and mortality worldwide. One of the most ominous manifestations of CHD is acute coronary syndrome (ACS), a life-threatening condition characterized by the rupture of the vulnerable atherosclerotic coronary plaque and subsequent thrombus formation, which leads to complete or incomplete occlusion of the coronary artery. ACS is the main cause of death in patients with CHD [1]. The ACS spectrum includes ST-segment elevation myocardial infarction (STEMI), non-STsegment elevation myocardial infarction (NSTEMI), and unstable angina pectoris (UAP). STEMI is the most severe form of ACS, characterized by complete occlusion of the infarct-related artery [2]. The incidence of STEMI varies worldwide. According to the European registry, the incidence of STEMI was estimated at about 44 to 142 per 100,000 population per year [3].

Primary percutaneous coronary intervention (PCI) is the gold standard for the management of STEMI, and it must be performed as soon as possible to restore the coronary flow, reduce the infarct area, and improve the prognosis [4]. Although primary PCI is effective in STEMI, some patients may still have unfavorable outcomes, including acute heart failure, reinfarction, stroke, repeat target vessel revascularization, and death [5]. Therefore, useful predictors are needed to help predict the adverse outcomes and guide appropriate follow-up and 
management. Some inflammatory biomarkers, such as C-reactive protein, interleukin-6, matrix metalloproteinase-9, and cystatin- $\mathrm{C}$ have been identified as useful predictors of adverse outcomes in patients with ACS, but these biomarkers are not widely available or used in clinical practice [6-9].

Platelets and leukocytes have vital roles in the pathogenesis of STEMI. Platelet activation plays a significant role in coronary thrombosis and occlusion after atherosclerotic plaque rupture. Elevated platelet count is associated with increased inflammation and platelet activation, which contributes to adverse outcomes in patients with STEMI $[10,11]$. In contrast, lymphocytes have a protective role in inflammation and atherosclerosis. Low lymphocyte count is associated with atherosclerosis progression and adverse outcomes in patients with STEMI [12]. The platelet-to-lymphocyte ratio (PLR), which is calculated by dividing the platelet counts by lymphocyte counts, has recently been proposed as a novel inflammatory biomarker and predictor of adverse outcomes in various cardiovascular diseases. PLR reflects both inflammation and thrombosis pathways and may be more valuable than either platelet or lymphocyte counts alone in predicting prognosis. PLR is a low-cost and widely available biomarker that can be a potential predictor in ACS $[13,14]$.

Several studies have found that elevated PLR is a predictor of poor prognosis in patients with ACS, including increased major adverse cardiac events (MACE) and mortality [15-17]. To our knowledge, there is a lack of meta-analysis that investigates the predictive value of PLR in the population of patients with STEMI undergoing primary PCI. Hence, we performed this meta-analysis to investigate the prognostic value of PLR as a predictor of in-hospital and long-term outcomes in patients with STEMI undergoing primary PCI.

\section{Materials and Methods}

\section{Search strategy}

This meta-analysis was conducted according to the Preferred Reporting Items for Systematic Reviews and Meta-Analyses (PRISMA) guidelines [18]. We performed a comprehensive systematic literature search in the PubMed, ScienceDirect, Cochrane Library, and ProQuest databases for eligible studies published up to December 2020. The keywords used were combination of "platelet-to-lymphocyte ratio", "platelet to lymphocyte ratio", "platelet lymphocyte ratio", "PLR", "acute coronary syndrome", "STEMI", and "percutaneous coronary intervention". There was no country or language restriction in the data search. In addition, the references of the relevant papers were also searched manually for potential additional articles.

\section{Inclusion and exclusion criteria}

The inclusion criteria for eligible studies were as follows: 1) cohort studies, either retrospective or prospective design; 2) patients diagnosed with STEMI and treated by primary PCI; 3) PLR was calculated at admission (preprocedure) before the primary PCI, with a cut-off value for defining high or low PLR; 4) reported data on in-hospital or long-term outcomes, including MACE and mortality, with outcomes comparison between low and high PLR group; and 5) available as full text. The exclusion criteria were as follows: 1) review articles, case reports, editorials, letters, and meeting abstracts; 2) non-human studies; and 3) studies with insufficient data for the estimation of odds ratios (ORs) and 95\% confidence intervals (CIs); and 4) overlapping or duplicate studies.

\section{Outcome of interest and definitions}

The outcomes were MACE and mortality, either in-hospital and long-term follow-up. In-hospital MACE was defined as any MACE that occurs during in-hospital period, including acute heart failure, re-infarction, malignant arrhythmia, stroke, the need for repeat target vessel revascularization, and cardiovascular death. In-hospital mortality was defined as any death, regardless of the cause, that occurs during in-hospital period. Long-term MACE was defined as any MACE that occurs during follow-up period after hospital discharge, including acute heart failure, re-infarction, malignant arrhythmia, stroke, the need for repeat target vessel revascularization, and cardiovascular death. Longterm mortality was defined as any death, regardless of the cause, that occurs during follow-up period after hospital discharge.

\section{Data extraction}

The data extraction from the relevant studies was performed independently by two authors (HAW and JCH). Any different decisions were resolved by discussion with the third author (HC) as supervisor. From the included studies, the data were extracted as follows: name of the first author, year of publication, country of origin, study design, sample size, mean age, proportion of male patients, duration of follow-up, PLR cut-off value, and outcome events.

\section{Quality assessment}

The included studies were independently assessed by two authors (HAW and JCH). Any different decisions were resolved once again by discussion with the third author (HC). The quality of each included study was systematically assessed using the Newcastle-Ottawa Scale (NOS) [19]. The total score of NOS was ranged from 0 to 9 based on the three aspects: selection (0 - 4 stars), comparability (0 - 2 stars), and outcome (0 - 3 stars). Studies with a total stars $\geq 7$ were considered as high quality studies, 5 - 6 were considered as moderate quality studies, and $\leq 4$ were considered as low quality studies.

\section{Statistical analysis}

This meta-analysis was performed using Review Manager 5.3 software (The Nordic Cochrane Centre, Copenhagen). The 


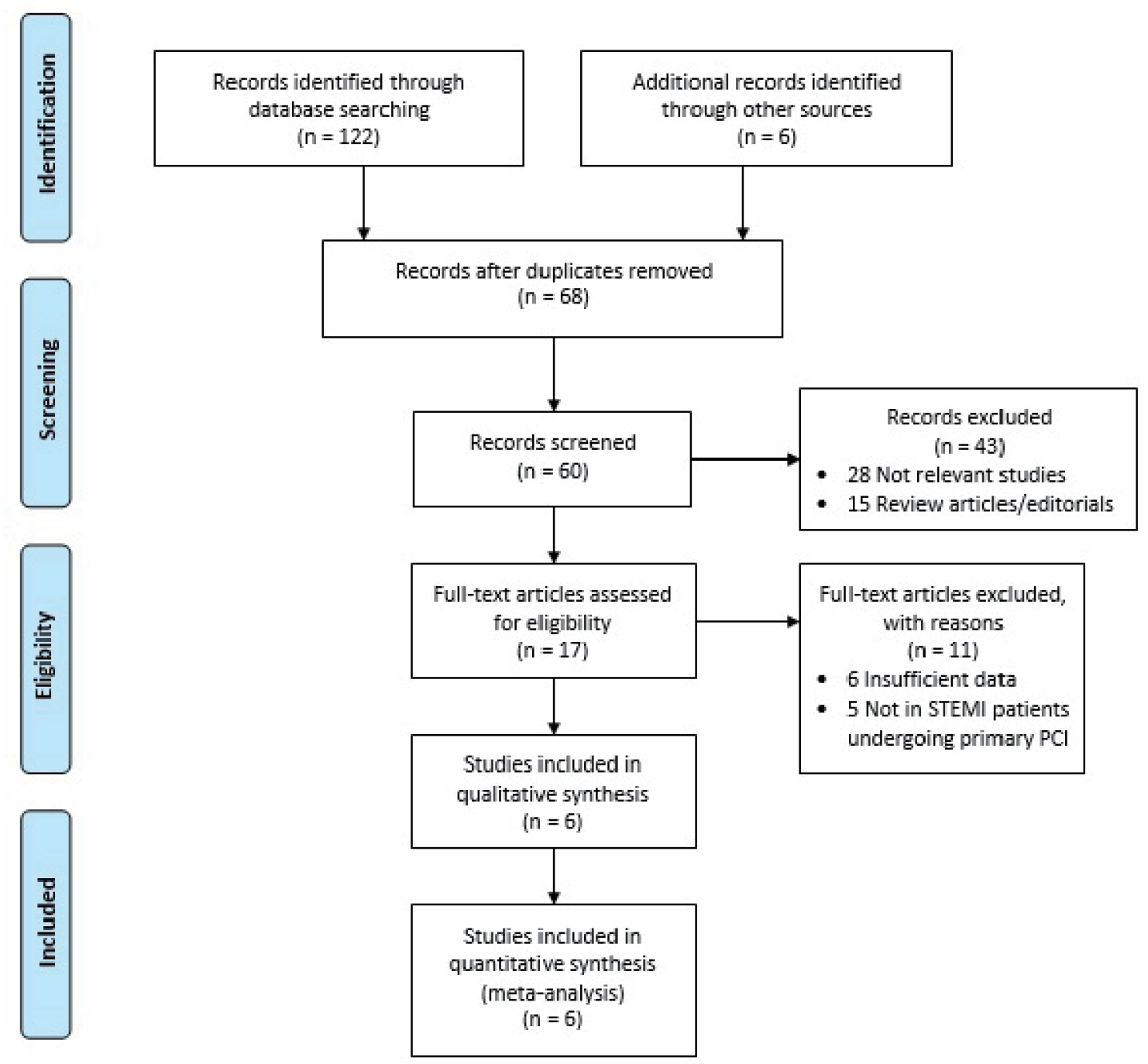

Figure 1. Literature search flow chart. PCI: percutaneous coronary intervention; STEMI: ST-segment elevation myocardial infarction.

ORs and its corresponding 95\% CI were calculated using the Mantel-Haenszel method to evaluate the association between admission PLR (high or low) and the clinical outcomes (inhospital MACE and mortality, as well as long-term MACE and mortality). The heterogeneity of the included studies was assessed using the Cochran's Q Chi-square test and $\mathrm{I}^{2}$ statistic. A fixed-effects model was used to calculate the pooled ORs and $95 \% \mathrm{CI}$ if $\mathrm{P} \geq 0.05$ and $\mathrm{I}^{2} \leq 50 \%$, which indicated no significant heterogeneity. If $\mathrm{P}<0.05$ or $\mathrm{I}^{2}>50 \%$, a random-effects model was used to calculate the pooled ORs because of substantial heterogeneity. A P value of $<0.05$ was considered as statistically significant for all test statistics. Potential publication bias was assessed using visual inspection of the funnel plots.

The Institutional Review Board approval was not required since this study is a systematic review and meta-analysis. This study was conducted in compliance with the ethical standards of the responsible institution on human subjects as well as with the Helsinki Declaration.

\section{Results}

\section{Literature search}

The systematic literature search from electronic databases identified an initial total of 122 potential articles, six of which were identified through manual hand-searching of relevant literatures. Following duplication removal, 60 articles were screened for the titles and abstracts. The remaining 17 articles were reviewed for the full text and 11 articles were excluded. Finally, six studies were included in our meta-analysis. Figure 1 shows the flow chart for the literature search process.

\section{Study characteristics and quality assessment}

There were six cohort studies with a total of 4,289 patients 
Table 1. Characteristics of the Studies Included in the Meta-Analysis

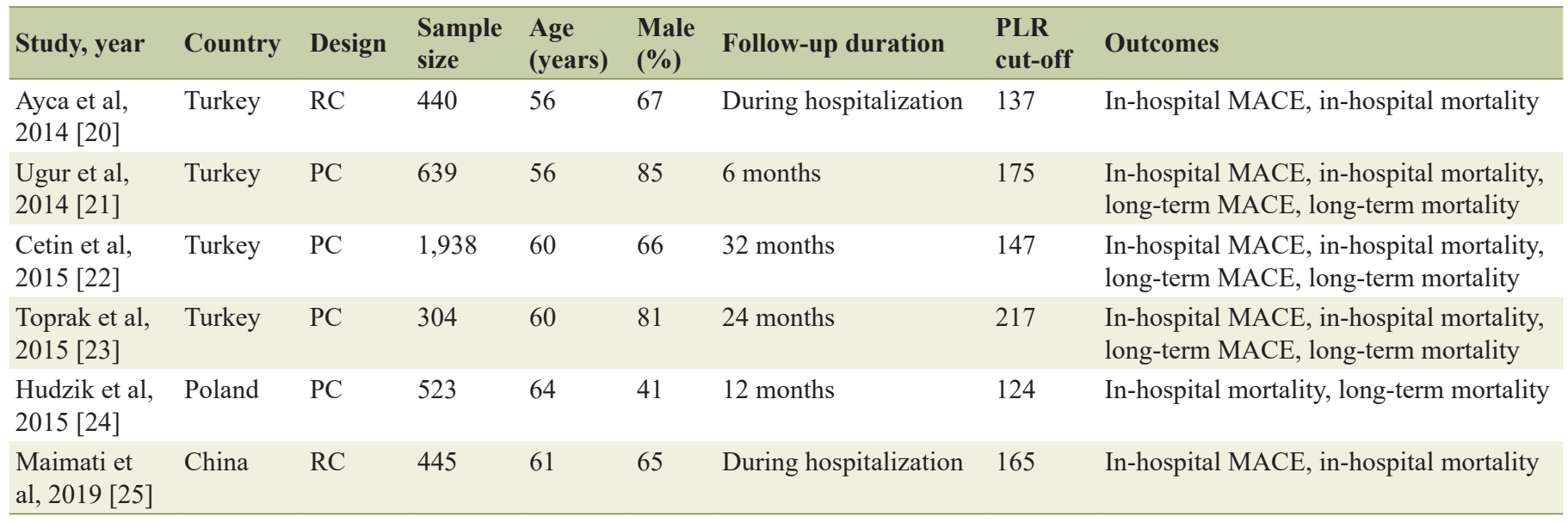

PLR: platelet-to-lymphocyte ratio; RC: retrospective cohort; PC: prospective cohort; MACE: major adverse cardiac events.

included in this meta-analysis [20-25]. The studies were published between 2014 and 2019. Two studies were retrospective cohort studies and four studies were prospective cohort studies. Four studies were carried out in Turkey [20-23], one in Poland [24], and one in China [25]. The mean age ranged from 56 to 64 years old. The follow-up duration ranged from during hospitalization to 32 months after discharged. The PLR cut-off values were varied and determined using different methods in each study, which ranged from 124 to 217 . One study determined the PLR cut-off value from the receiver operating characteristic analysis [20], whereas five studies determined the PLR cut-off value from the tertiles (third tertile versus lower tertile) [21-25]. The summary of the included studies are shown in Table 1 [2025]. For quality assessment of the included studies, we used the NOS. The NOS quality stars ranged from eight to nine stars, indicating generally high study quality (Table 2 [20-25]).

\section{Association between PLR and in-hospital outcomes in pa- tients with STEMI undergoing primary PCI}

The association between PLR and in-hospital MACE was reported in five studies, whereas the association between PLR and in-hospital mortality was reported in six studies. The pooled analysis with a fixed-effects model showed that compared to those with low PLR at admission, patients with high PLR had significantly increased risk of in-hospital MACE
$\left(\mathrm{OR}=1.94 ; 95 \% \mathrm{CI}=1.56-2.40 ; \mathrm{P}<0.00001 ; \mathrm{I}^{2}=45 \%\right)$ and in-hospital mortality $(\mathrm{OR}=2.07 ; 95 \% \mathrm{CI}=1.53-2.80 ; \mathrm{P}$ $\left.<0.00001 ; \mathrm{I}^{2}=50 \%\right)$. The forest plots are shown in Figure 2.

\section{Association between PLR and long-term outcomes in pa- tients with STEMI undergoing primary PCI}

The association between PLR and long-term MACE was reported in three studies, whereas the association between PLR and long-term mortality was reported in four studies. The pooled analysis with a random-effects model showed that compared to those with low PLR at admission, patients with high PLR had significantly increased risk of long-term MACE $\left(\mathrm{OR}=1.98 ; 95 \% \mathrm{CI}=1.31-3.00 ; \mathrm{P}=0.001 ; \mathrm{I}^{2}=72 \%\right)$ and long-term mortality $(\mathrm{OR}=2.79 ; 95 \% \mathrm{CI}=1.45-5.36 ; \mathrm{P}=$ $0.002 ; \mathrm{I}^{2}=83 \%$ ). The forest plots are shown in Figure 3 .

\section{Publication bias}

The funnel plot of the association of PLR with in-hospital MACE and in-hospital mortality showed symmetrical on inspection, suggesting low risk of publication bias (Fig. 4). The funnel plot of the association of PLR with long-term MACE and long-term mortality cannot be obtained due to the limited number of included studies, thus the possible publication bias

Table 2. Quality Assessment of the Included Studies by Newcastle-Ottawa Scale

\begin{tabular}{|c|c|c|c|c|}
\hline Study, year & Selection & Comparability & Outcome & Total rating \\
\hline Ayca et al, 2014 [20] & $\star \star \star \star \star$ & $\star \star$ & $\star \star$ & $8 \star$ \\
\hline Ugur et al, 2014 [21] & $\star \star \star \star ~$ & $\star \star$ & $\star \star \star \star$ & $9 \star$ \\
\hline Cetin et al, 2015 [22] & $\star \star \star \star ~$ & $\star \star$ & $\star \star \star$ & $9 \star$ \\
\hline Toprak et al, 2015 [23] & $\star \star \star \star \star ~$ & $\star \star$ & $\star \star \star \star$ & $9 \star$ \\
\hline Hudzik et al, 2015 [24] & $\star \star \star \star ~$ & $\star \star$ & $\star \star \star$ & $8 \star$ \\
\hline Maimati et al, 2019 [25] & $\star \star \star \star \star ~$ & $\star \star \star$ & $\star \star$ & $8 \star$ \\
\hline
\end{tabular}




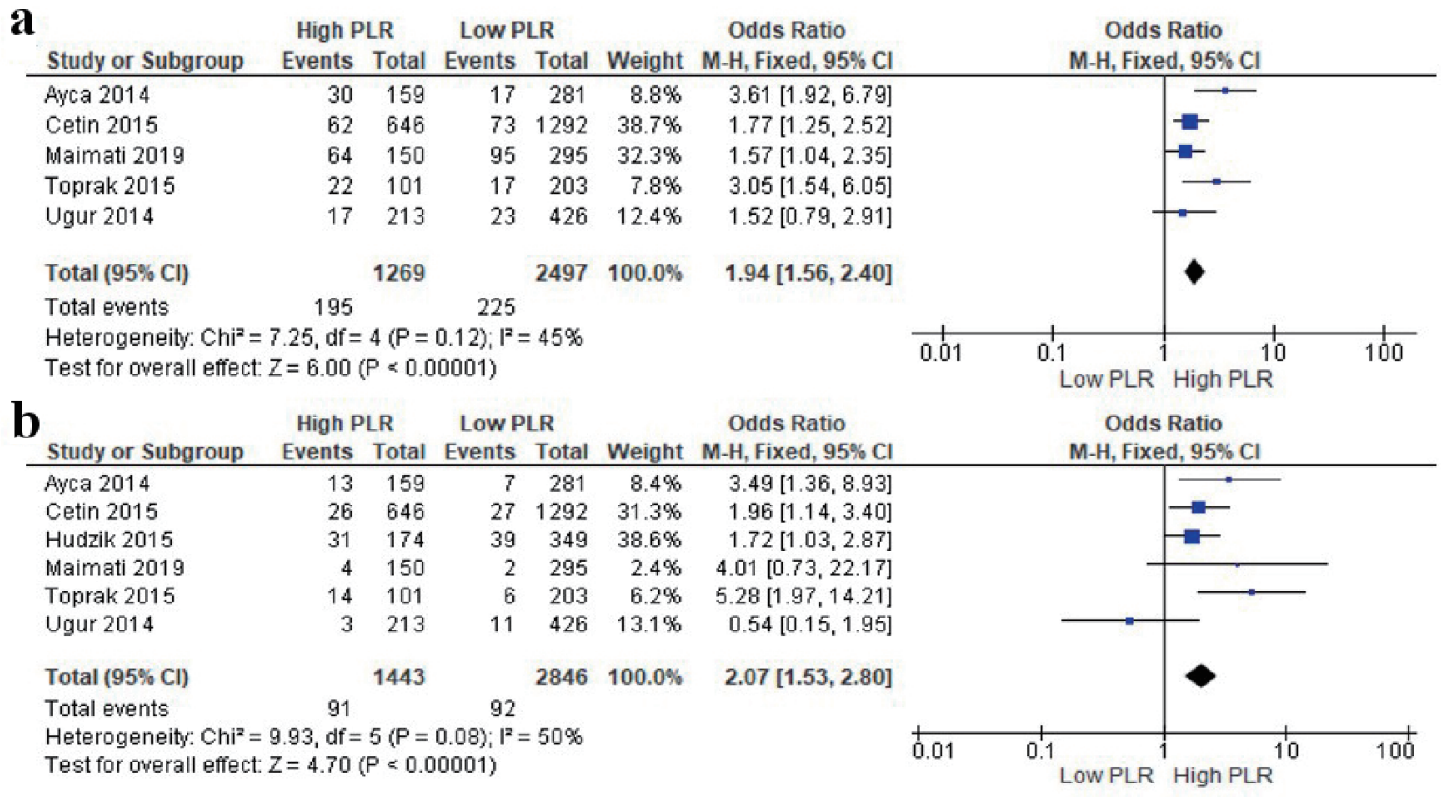

Figure 2. Forest plots of the association between PLR at admission and in-hospital outcomes in STEMI patients undergoing primary PCl. (a) In-hospital MACE. (b) In-hospital mortality. PLR: platelet-to-lymphocyte ratio; PCI: percutaneous coronary intervention; MACE: major adverse cardiac event; STEMI: ST-segment elevation myocardial infarction; Cl: confidence interval.

cannot be excluded.

\section{Discussion}

This systematic review and meta-analysis included six cohort studies involving a total of 4,289 STEMI patients treated by primary PCI. In pooled analysis we found that PLR was associated with both in-hospital and long-term outcomes. Compared to those with low PLR at admission, patients with high PLR had almost two-fold increased risk of in-hospital MACE, about two-fold increased risk of in-hospital mortality, almost

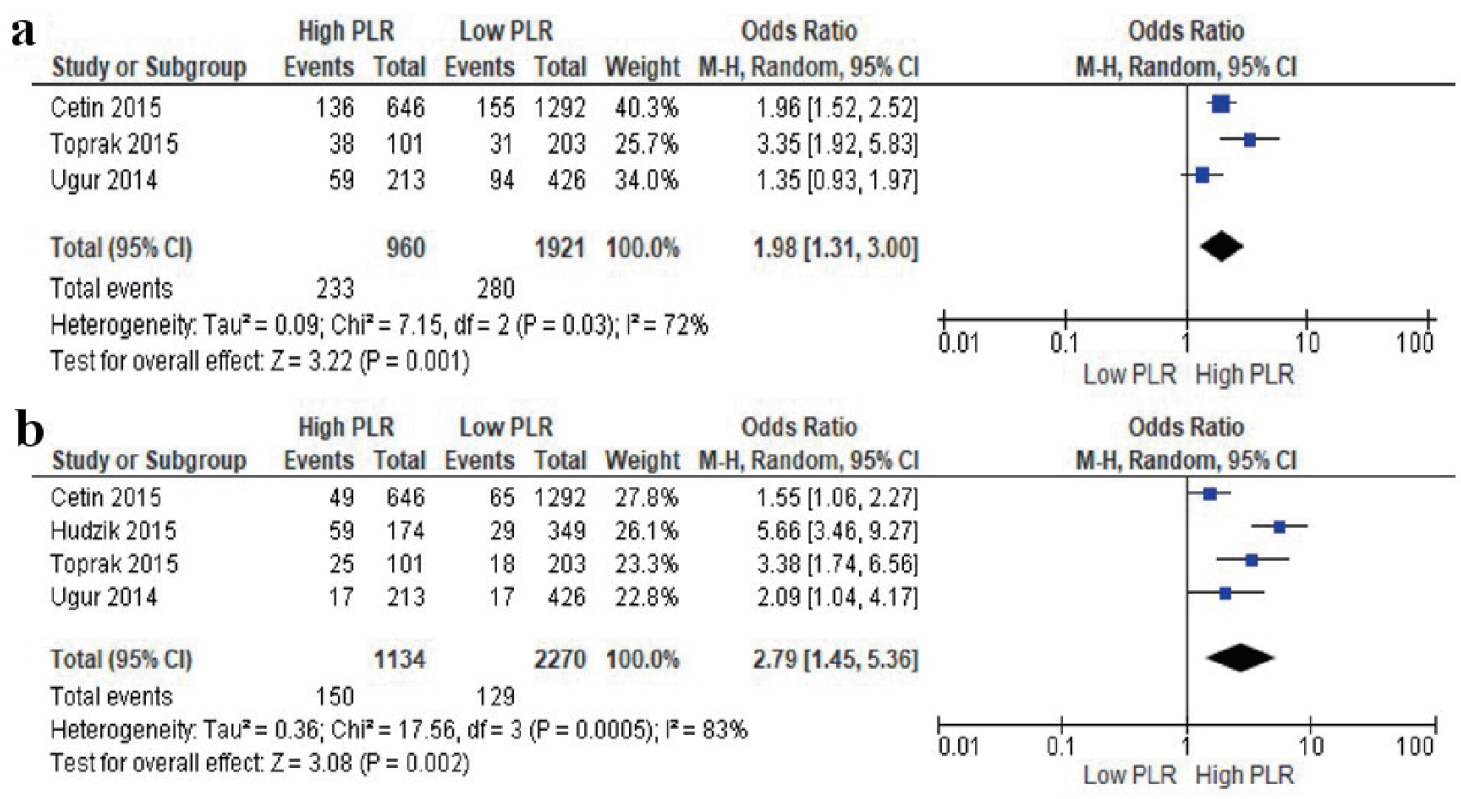

Figure 3. Forests plots of the association between PLR at admission and long-term outcomes in STEMI patients undergoing primary PCI. (a) Long-term MACE. (b) Long-term mortality. PLR: platelet-to-lymphocyte ratio; PCl: percutaneous coronary intervention; MACE: major adverse cardiac event; STEMI: ST-segment elevation myocardial infarction; Cl: confidence interval. 

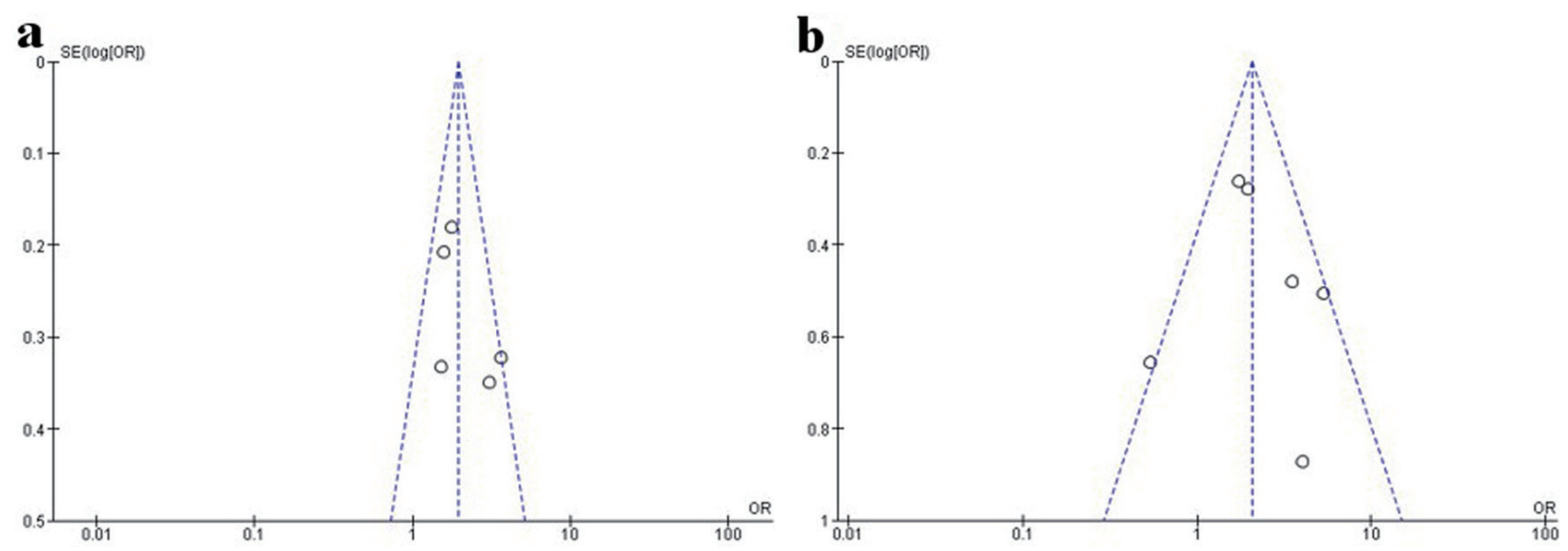

Figure 4. Funnel plots of the association between PLR at admission and in-hospital outcomes in STEMI patients undergoing primary PCl. (a) In-hospital MACE. (b) In-hospital mortality. PLR: platelet-to-lymphocyte ratio; PCl: percutaneous coronary intervention; MACE: major adverse cardiac event; STEMI: ST-segment elevation myocardial infarction.

two-fold increased risk of long-term MACE, and almost threefold increased risk of long-term mortality. Our results are similar with a meta-analysis by $\mathrm{Li}$ et al [26] involving a total of 6,627 patients with ACS, which demonstrated that high PLR was an independent predictor of in-hospital and long-term cardiovascular events and mortality. However, besides STEMI, Li et al also included patients with non-STEMI and unstable angina pectoris in their meta-analysis, either undergoing or not undergoing PCI [26]. In our meta-analysis, we only included patients with STEMI undergoing primary PCI.

Currently, in clinical practice, the Thrombolysis in Myocardial Infarction Risk (TIMI) score and Global Registry of Acute Coronary Events (GRACE) score are the most frequently used prognostic tools in STEMI [27]. Other predictors that are frequently used are Killip class and glomerular filtration rate [28]. We propose that PLR can be a potential prognostic tool and can be used in conjunction with other tools to improve prognostic value in STEMI patients undergoing primary PCI. However, the optimal cut-off of PLR for predicting prognosis still needs further investigation, since the cut-off values for defining high PLR were different among our included studies, which ranged from 124 to 217 .

The underlying mechanism by which a high PLR increases the risk of adverse outcomes in patients with STEMI is still not completely understood yet. A high PLR reflects inflammatory activity and prothrombotic status, which plays an important role in the pathogenesis of STEMI [29]. Platelets play a major role in all stages of atherosclerosis development and thrombus formation after atherosclerotic plaque destabilization. Rupture of the vulnerable plaque leads to local thrombin generation and fibrin deposition, which triggers platelet activation, adhesion, and aggregation, and leads to the formation of the intracoronary thrombus [30]. High platelet counts may reflect ongoing inflammation, as inflammatory mediators can stimulate megakaryocytic proliferation and cause relative thrombocytosis which contribute to a prothrombotic state [23]. Ly et al reported that higher platelet counts were associated with increased risk of adverse outcomes in patients with STEMI, including heart failure, re-infarction, and death [11]. Turakhia et al reported that high platelet counts were associated with the presence of residual thrombus after fibrinolytic therapy for STEMI [31]. Moreover, high platelet counts may reflect higher risk of antiplatelet drug resistance and higher tendency to form platelet-rich thrombus on the atherosclerotic plaques, which may lead to no-reflow phenomenon after primary PCI, as well as worse outcomes in patients with STEMI [24, 32].

In contrast to platelets, lymphocytes have anti-inflammatory properties and play a critical role in myocardial healing after acute myocardial infarction, especially T-lymphocytes. Lymphocytes can migrate to the inflamed myocardial tissues and secrete anti-inflammatory cytokines to limit the inflammatory responses $[33,34]$. Moreover, it was reported that in murine myocardial infarction models, infusion of T-lymphocytes can decrease infarction size and attenuate myocardial infarction-induced cardiac remodeling [35]. Lymphocyte counts are known to decrease during STEMI. Nunez et al reported that low lymphocyte counts in the first $96 \mathrm{~h}$ of STEMI predicted the risk of long-term recurrent myocardial infarction [12]. Low lymphocyte counts may reflect physiological stress response induced by the increased level of cortisol or catecholamines during ACS. Elevated cortisol and catecholamines may induce lymphocyte apoptosis and down-regulate proliferation and differentiation of lymphocyte [36].

As the PLR combines the predictive values of platelet and lymphocyte counts, it can be more valuable in predicting prognosis than platelet or lymphocyte counts alone. Considering the findings of our meta-analysis that PLR was associated with in-hospital and long-term outcomes, we suggest that PLR can be used as a routine prognostic biomarker to help risk stratification of STEMI patients who undergo primary PCI and guide the appropriate management. Patients who have a high PLR at admission can be considered as high-risk patients and require more intensive therapy and follow-up.

\section{Study limitations}

There were some limitations in our meta-analysis. First, most 
studies included in our meta-analysis were conducted in Turkey, which may not represent the global population. Second, the number of included study is relatively small because of limited data availability. There is still a limited number of research that reported the association of PLR at admission with in-hospital and long-term outcomes in the population of STEMI patients undergoing primary PCI. Third, the cut-off values for determining high and low PLR were varied among included studies. Further studies are needed to determine the optimal cut-off value of PLR. Fourth, substantial heterogeneity was found in the long-term MACE and long-term mortality. We did not get enough data for subgroup analysis. Fifth, the funnel plot of long-term MACE and long-term mortality cannot be obtained due to limited number of included studies, thus the possible publication bias cannot be excluded.

\section{Conclusions}

This systematic review and meta-analysis showed that in patients with STEMI undergoing primary PCI, a high PLR at admission predicts in-hospital MACE and mortality, as well as long-term MACE and mortality. Considering that PLR is a simple and easily obtainable biomarker from routine laboratory test, we suggest PLR can be used routinely along with other clinical biomarkers to guide prognostic assessment and follow-up in STEMI patients undergoing primary PCI.

\section{Acknowledgments}

We would like to thank to all our team and family members whose prayers and supports made it possible to finish this manuscript.

\section{Financial Disclosure}

The authors received no financial support for the research or publication of this manuscript.

\section{Conflict of Interest}

The authors have no conflict of interest in the publication of this manuscript.

\section{Informed Consent}

Not applicable.

\section{Author Contributions}

HAW and JCH contributed in study concept and design, systematic literature search, data analysis, statistical analysis, and writing the manuscript. HC contributed in supervision and re- vision of manuscript for critically important intellectual content. All authors have approved the final version of the manuscript.

\section{Data Availability}

The authors declare that data supporting the findings of this study are available within the article.

\section{References}

1. Bentzon JF, Otsuka F, Virmani R, Falk E. Mechanisms of plaque formation and rupture. Circ Res. 2014;114(12):1852-1866.

2. Paul K, Mukherjee S, Ghosh S, Mandal MS. Evaluation and outcome of patients of STEMI with acute total occlution of coronary artery in the setting of primary PCI, pharmaco invasive PCI and delayed PCI. J Cardiol Cardiovasc Ther. 2018;12(5):1-6.

3. Widimsky P, Wijns W, Fajadet J, de Belder M, Knot J, Aaberge L, Andrikopoulos G, et al. Reperfusion therapy for ST elevation acute myocardial infarction in Europe: description of the current situation in 30 countries. Eur Heart J. 2010;31(8):943-957.

4. Nallamothu BK, Normand SL, Wang Y, Hofer TP, Brush JE, Jr., Messenger JC, Bradley EH, et al. Relation between door-to-balloon times and mortality after primary percutaneous coronary intervention over time: a retrospective study. Lancet. 2015;385(9973):1114-1122.

5. Ayad SW, Sobhy M, Zaki A, Elkammash A. Predictive factors of in hospital major adverse cardiac events and no reflow phenomenon in patients with ST elevation myocardial infarction undergoing primary percutaneous coronary intervention. J Integr Cardiol. 2015;1(4):104-106.

6. Sheikh AS, Yahya S, Sheikh NS, Sheikh AA. C-reactive protein as a predictor of adverse outcome in patients with acute coronary syndrome. Heart Views. 2012;13(1):7-12.

7. Li H, Cen K, Sun W, Feng B. Predictive Value of Blood Interleukin-6 Level in Patients with Acute Coronary Syndrome: A Meta-analysis. Immunol Invest. 2020:1-13.

8. Lahdentausta L, Leskela J, Winkelmann A, Tervahartiala T, Sorsa T, Pesonen E, Pussinen PJ. Serum MMP-9 diagnostics, prognostics, and activation in acute coronary syndrome and its recurrence. J Cardiovasc Transl Res. 2018;11(3):210-220.

9. Correa S, Morrow DA, Braunwald E, Davies RY, Goodrich EL, Murphy SA, Cannon CP, et al. Cystatin C for risk stratification in patients after an acute coronary syndrome. J Am Heart Assoc. 2018;7(20):e009077.

10. Stakos DA, Tziakas DN, Stellos K. Mechanisms of platelet activation in acute coronary syndromes. Curr Vasc Pharmacol. 2012;10(5):578-588.

11. Ly HQ, Kirtane AJ, Murphy SA, Buros J, Cannon CP, Braunwald E, Gibson CM, et al. Association of platelet counts on presentation and clinical outcomes in STelevation myocardial infarction (from the TIMI Trials). Am J Cardiol. 2006;98(1):1-5. 
12. Nunez J, Nunez E, Bodi V, Sanchis J, Mainar L, Minana $\mathrm{G}$, Facila L, et al. Low lymphocyte count in acute phase of ST-segment elevation myocardial infarction predicts long-term recurrent myocardial infarction. Coron Artery Dis. 2010;21(1):1-7.

13. Kurtul A, Ornek E. Platelet to Lymphocyte Ratio in Cardiovascular Diseases: A Systematic Review. Angiology. 2019;70(9):802-818.

14. Budzianowski J, Pieszko K, Burchardt P, Rzezniczak J, Hiczkiewicz J. The Role of Hematological Indices in Patients with Acute Coronary Syndrome. Dis Markers. 2017;2017:3041565.

15. Oylumlu M, Yildiz A, Oylumlu M, Yuksel M, Polat N, Bilik MZ, Akyuz A, et al. Platelet-to-lymphocyte ratio is a predictor of in-hospital mortality patients with acute coronary syndrome. Anatol J Cardiol. 2015;15(4):277283.

16. Kurtul A, Murat SN, Yarlioglues M, Duran M, Ergun G, Acikgoz SK, Demircelik MB, et al. Association of platelet-to-lymphocyte ratio with severity and complexity of coronary artery disease in patients with acute coronary syndromes. Am J Cardiol. 2014;114(7):972-978.

17. Azab B, Shah N, Akerman M, McGinn JT, Jr. Value of platelet/lymphocyte ratio as a predictor of all-cause mortality after non-ST-elevation myocardial infarction. J Thromb Thrombolysis. 2012;34(3):326-334.

18. Moher D, Liberati A, Tetzlaff J, Altman DG, The PRISMA Group. Preferred reporting items for systematic reviews and meta-analyses: the PRISMA statement. PLoS Med. 2009;6(7):e1000097.

19. Wells GA, Shea B, O'Connel D, Peterson J, Welch V, Losos M, Tugwell P. The Newcastle-Ottawa Scale (NOS) for assessing the quality of nonrandomised studies in meta-analyses [Internet]. 2011 [cited Dec 28, 2020]. Available from: http://www.ohri.ca/programs/clinical_epidemiology/oxford.asp.

20. Ayca B, Akin F, Celik O, Yuksel Y, Ozturk D, Tekiner F, Cetin S, et al. Platelet to lymphocyte ratio as a prognostic marker in primary percutaneous coronary intervention. Platelets. 2015;26(7):638-644.

21. Ugur M, Gul M, Bozbay M, Cicek G, Uyarel H, Koroglu $\mathrm{B}$, Uluganyan $\mathrm{M}$, et al. The relationship between platelet to lymphocyte ratio and the clinical outcomes in ST elevation myocardial infarction underwent primary coronary intervention. Blood Coagul Fibrinolysis. 2014;25(8):806811.

22. Ozcan Cetin EH, Cetin MS, Aras D, Topaloglu S, Temizhan A, Kisacik HL, Aydogdu S. Platelet to lymphocyte ratio as a prognostic marker of in-hospital and long-term major adverse cardiovascular events in ST-segment elevation myocardial infarction. Angiology. 2016;67(4):336-345.

23. Toprak C, Tabakci MM, Simsek Z, Arslantas U, Durmus HI, Ocal L, Demirel M, et al. Platelet/lymphocyte ratio was associated with impaired myocardial perfusion and both in-hospital and long-term adverse outcome in patients with ST-segment elevation acute myocardial infarction undergoing primary coronary intervention. Postepy Kardiol Interwencyjnej. 2015;11(4):288-297.
24. Hudzik B, Szkodzinski J, Gorol J, Niedziela J, Lekston A, Gasior M, Polonski L. Platelet-to-lymphocyte ratio is a marker of poor prognosis in patients with diabetes mellitus and ST-elevation myocardial infarction. Biomark Med. 2015;9(3):199-207.

25. Maimaiti A, Li Y, Wang YT, Yang X, Li XM, Yang YN, Ma YT. Association of platelet-to-lymphocyte count ratio with myocardial reperfusion and major adverse events in patients with acute myocardial infarction: a two-centre retrospective cohort study. BMJ Open. 2019;9(9):e025628.

26. Li H, Zhou Y, Ma Y, Han S, Zhou L. The prognostic value of the platelet-to-lymphocyte ratio in acute coronary syndrome: a systematic review and meta-analysis. Kardiol Pol. 2017;75(7):666-673.

27. Correia LC, Garcia G, Kalil F, Ferreira F, Carvalhal M, Oliveira R, Silva A, et al. Prognostic value of TIMI score versus GRACE score in ST-segment elevation myocardial infarction. Arq Bras Cardiol. 2014;103(2):98-106.

28. Ginanjar E, Yamin M, Wijaya IP, Harimurti K. Predictors of 30-day Mortality in ST-Elevation Myocardial Infarction (STEMI) Patients. Acta Med Indones. 2019;51(3):238-244.

29. Kounis NG, Koniari I, Plotas P, Soufras GD, Tsigkas G, Davlouros P, Hahalis G. Inflammation, thrombosis, and platelet-to-lymphocyte ratio in acute coronary syndromes. Angiology. 2021;72(1):6-8.

30. Kaminska J, Koper OM, Siedlecka-Czykier E, Matowicka-Karna J, Bychowski J, Kemona H. The utility of inflammation and platelet biomarkers in patients with acute coronary syndromes. Saudi J Biol Sci. 2018;25(7):12631271.

31. Turakhia MP, Murphy SA, Pinto TL, Antman EM, Giugliano RP, Cannon CP, Braunwald E, et al. Association of platelet count with residual thrombus in the myocardial infarct-related coronary artery among patients treated with fibrinolytic therapy for ST-segment elevation acute myocardial infarction. Am J Cardiol. 2004;94(11):14061410 .

32. Ali GMS, Dimitry SR, Helmy HAR, Ahmed MA. Assessment of role of platelet-to-lymphocyte ratio in prediction of angiographic no-reflow in patients subjected to primary percutaneous coronary intervention. OJCHD. 2020;3(2):1-7.

33. Hofmann U, Frantz S. Role of lymphocytes in myocardial injury, healing, and remodeling after myocardial infarction. Circ Res. 2015;116(2):354-367.

34. Wang YP, Xie Y, Ma H, Su SA, Wang YD, Wang JA, Xiang MX. Regulatory T lymphocytes in myocardial infarction: A promising new therapeutic target. Int J Cardiol. 2016;203:923-928.

35. Matsumoto K, Ogawa M, Suzuki J, Hirata Y, Nagai R, Isobe M. Regulatory T lymphocytes attenuate myocardial infarction-induced ventricular remodeling in mice. Int Heart J. 2011;52(6):382-387.

36. Bian C, Wu Y, Shi Y, Xu G, Wang J, Xiang M, Weng S, et al. Predictive value of the relative lymphocyte count in coronary heart disease. Heart Vessels. 2010;25(6):469473. 